\title{
Il filosofo platonico secondo Damascio Franco Trabattoni
}

1.

Inizierò rammentando alcuni fatti ben noti a proposito del testo che qui ci interessa, ossia della cosiddetta Vita Isidori, scritta dal filosofo neoplatonico, nonché ultimo scolarca dell'accademia, Damascio di Damasco. Lo stato in cui quest'opera ci è pervenuta è frammentario e fino alle edizioni ottocentesche si riteneva che l'unica fonte per ricostruirla (una fonte, peraltro, abbastanza copiosa) fossero gli estratti contenuti nel cod. 242 della Biblioteca di Fozio (a cui si può aggiungere quelli specie di recensione del libro, per usare la felice espressione di Athanassiadi ${ }^{1}$, preservata nel cod. 181). Agli inizi del ' 900 lo studioso tedesco Rudolph Asmus pubblicò alcuni lavori sull'argomento ${ }^{2}$, culminati in una edizione completa dell'opera nella sola lingua tedesca (1913), in cui ricostruiva lo scritto combinando i codici di Fozio menzionati con alcune voci della Suda ${ }^{3}$. Molti anni dopo (1967) C. Zintzen pubblicò il testo greco ricalcando senza innovare nulla la ricostruzione di Asmus ${ }^{4}$. Infine, nel 1999 Polymnia Athanassiadi pubblicava un'edizione dell'opera in cui rigettava l'impostazione generale adottata da Asmus e Zintzen, che a suo avviso tradiva lo spirito del testo concentrando il focus dello scritto sulla sola biografia di Isidoro, quando invece esso possiede a suo avviso un ben più ampio respiro; e questa differenza era resa immediatamente visibile anche dal fatto che mentre Asmus e Zintzen avevano ipotizzato il titolo sulla base di quanto ne dice Fozio (che parla appunto di un libro che ha per argomento La vita di Isidoro), Athanassiadi preferisce adottare il titolo che si legge nella Suda, ossia Storia filosofica.

In realtà la questione è straordinariamente complessa, e temo che non consenta di pervenire a conclusioni sicure. Da un lato il fatto che il testo, almeno nelle condizioni in cui è giunto a noi, contenga molto di più che la biografia del solo Isidoro è del tutto evidente. Inoltre ciò è confermato da quanto si legge nel cod. 181 di Fozio, laddove il patriarca scrive che Damascio "ha scritto non più la vita di Isidoro che quella di molti altri, sia suoi contemporanei sia predecessori, raccogliendo fatti e racconti che li riguardano, e facendo sovente un uso anche eccessivo della digressione" (126a8-12). D'altra parte gli estratti prodotti da Fozio sono talvolta così sintetici e tra loro slegati (ma forse non proprio, come ancora osserva Athanassiadi, "giustapposti in forma anarchica",5), che risulta davvero difficile ricostruire uno sviluppo organico dell'opera; e dunque anche decidere quanto fosse centrale in essa la biografia di Isidoro e quanto eccentriche, ovvero funzionali al supposto obiettivo d'insieme, fossero le digressioni. E neppure è facile capire che cosa intenda Fozio per "digressione", dal momento che ciò avrebbe senso solo se l'opera avesse un nucleo centrale, ossia la biografia di Isidoro; ma se questo nucleo non c'è, la digressione è digressione da che cosa? Più in generale, l'annotazione di Isidoro che abbiamo appena citato a mio avviso non deve essere intesa tanto come un'osservazione oggettiva, quanto come l'iperbole critica di chi rileva con fastidio che spesso e volentieri l'autore sembra abbandonare l'intento generale dell'opera; che però questa stessa critica ha l'effetto di confermare. In proposito c'è il significativo precedente di Eunapio, che riempie le sue Vite di digressioni su personaggi diversi dal protagonista (si v. ad es. la vita di Edesio), e oltretutto giustifica esplicitamente la pertinenza di questa sua scelta (cfr. VI, 2, 12).

Quello che voglio dire, in definitiva, è che da un lato la complessità della Vita Isidori non esclude che l'essenza dello scritto sia di tipo biografico (e che dunque le finalità dell'opera siano quelle tipiche di questo genere letterario); e dall'altro che la preminenza del tema biografico non può far

\footnotetext{
${ }^{1}$ Athanassiadi 1999, p. ***

${ }^{2}$ Asmus 1909/10 e 1910.

${ }^{3}$ Asmus 1913.

${ }^{4}$ Zintzen 1967.

${ }^{5}$ Athanassiadi 1999, p. ***
} 
passare sotto silenzio il fatto che questo scritto non è soltanto una biografia, del tipo di quelle di Porfirio o di Marino. Perciò, a mio parere, il dissenso tra Athannsiadi e Asmus si risolve, da un certo punto di vista, senza né vinti né vincitori: se è vero che la ricostruzione di Asmus è caratterizzata dal fatto di raggruppare il materiale intorno ad Isidoro (At. 63), sulla base dell'idea preconcetta che la biografia di questo personaggio sia il tema centrale dello scritto, la nuova edizione di Athanassiadi, benché all'atto pratico sia più fedele all'ordine stabilito da Asmus di quanto in teoria si potrebbe supporre, talvolta se ne discosta in base al pregiudizio esattamente contrario: ossia che lo scritto di Damascio sia essenzialmente cosa diversa dalla biografia di Isidoro. Sia come sia, la questione più interessante non è probabilmente questa. Indipendentemente dallo spazio grande o piccolo occupato da Isidoro nel testo, qual è il rapporto di quest'opera con la tradizione delle biografia filosofica antica (e della biografia in generale)? Anzitutto osserviamo un fatto curioso, notato a suo tempo da Arnaldo Momigliano ${ }^{6}$ (e oggi confermato dagli strumenti elettronici): nella Vita Isidori si trova l'unica occorrenza, in tutta la letteratura greca, del termine

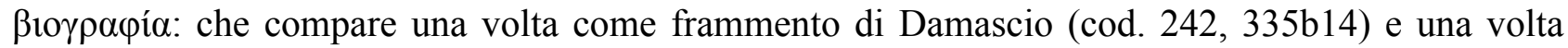
come ripresa da parte di Fozio (cod. 181, 126a5). Dunque, anche solo per questa specie di autotestimonianza sembra difficile separare il libro di Damascio dalla tradizione biografica.

Tale è invece, come detto, l'opinione della Athanassiadi. A suo parere la vera intenzione di Damascio era quella di comporre, cito, una "great work of trascendental propsopography" (58); ma per venire in contro alle richieste di Teodora, che era stata discepola sia sua che di Isidoro, e per dimostrare un rispetto formale alla tradizione (Porfirio e Plotino; Marino e Proclo, ecc.), Damascio avrebbe deciso di dare al suo lavoro il titolo alternativo Vita di Isidoro. Quanto al genere letterario proprio dell'opera, sempre secondo Athanassiadi è da scartare sia il confronto con la Storia filosofica progettata da Porfirio (di cui possediamo solo l'introduzione, ossia la Vita di Pitagora) sia quello con le Vite di Eunapio. Nel primo caso ciò che osta è il carattere compilatorio ed antiquario dello scritto di Porfirio. Nel secondo il fatto che mentre le vite di Eunapio hanno un tono assolutamente agiografico, e dunque sono sprovviste di qualunque spunto critico, il libro di Damascio è talmente duro nei confronti dei personaggi che cita, compresi quelli che sono lodati ed anche lo stesso Isidoro, da suscitare la reazione stizzita di Fozio (di questo parleremo più avanti). Perciò, prosegue Athanassiadi, l'unica opera del passato che può costituire un precedente a quella di Damascio è il libro perduto di Numenio Sul dissenso tra accademici e platonici (***). A suo parere, infatti, entrambi questi lavori contengono una critica al passato mirata a ricostruire il futuro su nuove basi. Detto questo (il che, come vedremo, è abbastanza plausibile, ma non dirime il rapporto tra la Vita Isidori e il genere biografico) Athanassiadi prosegue sostenendo che l'opera di Damascio anticipa in molti modi la Recherche proustiana (***): la Storia filosofica sarebbe la descrizione di un piccolo gruppo di persone, dei cerchi concentrici che in vario modo li hanno contenuti, e delle linee che li hanno interessati tangenzialmente. In termini più concreti, secondo questa studiosa Damascio sarebbe, in analogia con Ronald Syme "lo storico sociale del platonismo tardo-antico" (41), in quanto non si limita a parlare degli individui ma chiama in causa "dinastie filosofiche", mostrando come la loro ascesa e il loro declino modifichino il panorama sociale dell'antichità.

Se prescindiamo dall'azzardato e sorprendente paragone con Proust, l'ipotesi di Athanassiadi che abbiamo sinteticamente riassunto contiene molti elementi di verità. Ma non vedo come questo sia sufficiente per isolare lo scritto di Damascio dalla tradizione biografica a cui indubbiamente appartiene. La stessa Athanassiadi riconosce alla Vita un intento moraleggiante che invece non si riscontra nell'opera di Syme; ma questo intento moraleggiante è conseguito soprattutto (se non esclusivamente) attraverso il carattere paradigmatico, se non proprio agiografico, che è tipico di tanta parte della letteratura biografica antica. E' certamente utile tenere conto del fatto che Damascio abbia spesso un atteggiamento critico, talvolta anche nei confronti di personaggi che sono per lo più oggetto delle sue lodi. In un passo molto citato (Fozio 181, 126a18-30) Fozio così si esprime:

\footnotetext{
${ }^{6}$ Momigliano 1974, 14.
} 
Di tutti coloro che esalta con le sue parole, e celebra come divenuti superiori alla natura umana sia per aver conseguito le più elevate conoscenze scientifiche sia per la celerità del pensiero, di ciascuno di essi erge se stesso a giudice, e non c'è nessuno di coloro che ammira a cui non rimproveri qualche insufficienza: quello che è apprezzato per la sua intelligenza, non è completamente intelligente; quello che è incomparabile per la sia sapienza non è del tutto sapiente; quello che è prossimo a dio per la sua virtù ha anche parecchi difetti. Così, ciascuno di quelli che porta in alto con le sue lodi è anche schernito e gettato a terra; in questo modo egli decreta in maniera indiretta la sua superiorità su tutti costoro in qualunque ambito.

In realtà ritengo che questo giudizio di Fozio abbia influenzato in modo eccessivo gli studiosi moderni. La stessa Athanassiadi, pur mettendo in luce una serie di ragioni (assolutamente plausibili) che spiegano l'atteggiamento polemico di Damascio, non rinuncia del tutto alla spiegazione "psicologica" tradizionale, secondo cui il diadoco avrebbe avuto una natura ipercritica e irascibile (26). Ma se leggiamo quanto dice in proposito Richard Goulet, in un articolo apparso quasi contemporaneamente al libro dell'Athanassiadi, la faccenda sembra molto diversa:

Lorsqu'on lit les Vies des Sophistes de Philostrate, les Vies d'Eunape on encore les fragments de la Vie d'Isidore de Damascius, on est frappé de constater la part qui y est faite à la critique de ces sophistes ou de ces philosophes. C'est que ces biographies ne sont pas écrites par des historiens indépendants, mais par des collègues dans la profession pour qui la critique des contemporains et des devanciers est une activité indispensable. $^{7}$

A parere di Goulet non esiste dunque tra le Vite di Eunapio (e ricordiamo che lo studioso francese è uno dei massimi esperti di questo autore, nonché in generale della tradizione biografica tardo antica $^{8}$ ) e la Vita di Damascio quella differenza così marcata di tono (agiografico il primo testo, polemico e malevolo il secondo) su cui la Athanassiadi fonda la tesi secondo cui i due scritti apparterrebbero a generi letterari diversi.

Detto questo, io credo che il commento di Fozio, se rettamente inteso, ci fornisca un'interessante chiave di lettura per comprendere lo scopo della Vita Isidori nel suo complesso. Se è vero che l'intento coperto di Damascio è quello di lodare se stesso, il retroscena dell'opera potrebbe essere quello che io avevo supposto ormai quasi trent'anni $\mathrm{fa}^{9}$ (e che ha trovato qualche benevolo riscontro da parte dl Philippe Hoffmann ${ }^{10}$ ): con la Vita Isidori Damascio promuove la legittimità dell'innesto, nell'Accademia morente, sullo scorcio del V secolo, di intellettuali provenienti da Alessandria, e in particolare l'opportunità e legittimità della sua diadochia. In altre parole - anche se qui non ho intenzione di entrare nel dettaglio di quella vecchia idea - nella Vita Isidori Damascio potrebbe avere l'intenzione di giustificare a posteriori tutta una lunga vicenda di politica, intellettuale e accademica iniziata con il primo viaggio di Isidoro ad Atene. In tal modo Damascio otteneva un duplice scopo: lodando Isidoro, lodava soprattutto la tradizione a cui questi, non meno che lui stesso, apparteneva; criticandolo in parte, mostrava che il frutto migliore di quella tradizione era proprio lui stesso. Quello che avrei da aggiungere oggi, alla luce di una più profonda conoscenza dei commentari platonici di Damascio, è che forse quest'ultimo non esentava di colpe, in merito alla decadenza scientifica dell'accademia, lo stesso Proclo.

\section{2.}

Non ho tuttavia intenzione, come detto, di insistere su questo punto. Nella seconda sezione di questo intervento vorrei piuttosto tentare una breve analisi della parte dell'opera dedicata

\footnotetext{
${ }^{7}$ Goulet 1998, 230-31.

${ }^{8}$ Goulet 2001 .

${ }^{9}$ Trabattoni 1985.

${ }^{10}$ Hoffmann 1994, p.
} 
specificamente alla vita di Isidoro, per mostrare da un lato qual è il tipo di modello di filosofo platonico che Damascio ha in mente, dall'altro che a questo tipo di filosofo appartengono tratti generali non necessariamente connessi con la particolare individualità della persona, ed anche compatibili con elementi di carattere ordinario o non eccelso. Quello che voglio dire è che l'intento di Damascio non è tanto quello di celebrare le eccezionali ed irripetibili qualità intellettuali ed umane del suo maestro (più o meno come avevano fatto Porfirio con Plotino e Marino con Proclo), quanto piuttosto quello di illustrare, contrapponendo esempi positivi ad esempi negativi, ciò che dovrebbe essere in generale un filosofo platonico all'altezza del suo compito e delle sue responsabilità.

Lo scopo generale di questa impresa, d'altra parte, non è troppo difficile da capire. Per salvare la grande tradizione della filosofia greco-pagana dall'egemonia sempre più intollerante del cristianesimo non bastava certo l'apporto di una o più personalità eccezionali (come aveva ben mostrato il caso di Giuliano); occorreva invece contrapporre alla ormai fiorente tradizione della chiesa romana, e alla sua capacità di perpetuarsi e di espandersi, una tradizione altrettanto forte, e dunque precisare quali erano in generale i requisiti e le condizioni necessari alla riproduzione, e alla continuazione nel tempo, del sapere filosofico antico. La stessa successione alla diadochia ateniese diviene in un certo senso il simbolo di questa continuità. I Cristiani ritenevano di essere in grado, grazie all'assistenza inesauribile dello Spirito Santo, di reintegrare ad libitum la comunità di coloro che erano a un tempo depositari del sapere sacro e guide per il popolo. In questo modo essi sembravano aver realizzato, proprio grazie all'assistenza soprannaturale, il sogno platonico di programmare l'indefinita riproducibilità tecnica (per usare un'espressione di Benjamin) dei sapienti - un sogno che in Platone si era infranto proprio davanti all'inevitabile imperfezione della ragione umana e all'inesistenza dell'aiuto soprannaturale. Come fra poco vedremo, il filosofo platonico che ha in mente Damascio obbedisce esattamente a questo modello, perché se da un lato appare solidamente coinvolto nell'uso dialettico della ragione e nelle faccende di questo modo, dall'altro deriva la sua autorità proprio da un contatto privilegiato con il soprannaturale. Ma se è vero, come abbiamo suggerito, che il problema decisivo per Damascio è proprio quello "platonico" della riproducibilità del filosofo, ben si capisce perché Isidoro non occupi tutta la scena: è interesse preciso di Damascio mostrare che i destini della filosofia platonica non dipendono dalla fortuna di imbattersi in un personaggio eccezionale come Isidoro, per quanto da lui esaltato in modo particolare per ragioni ben comprensibili: se le condizioni naturali sono quelle giuste, e gli orientamenti intellettuali e etici sono quelli corretti, dopo Isidoro ci potrà essere un Damascio, e così via nel futuro.

Iniziamo la nostra analisi da due frammenti di Fozio $(242,7-8)$ e da un paio di righe della Suda (III, 35. 30), che Athanassiadi ha ragionevolmente giustapposto. Nel fr. 7 di Fozio si legge che "essa “ (presumibilmente l'anima di Damascio), essendo precipitata dalla volta celeste è legata alla vita terrena". Analogo il passo della Suda. Qui si dice che "egli", evidentemente Damascio, nel momento di discendere nella generazione abbia proclamato: "Ma io giungo qui da un luogo migliore". Nel frammento 8 di Fozio si legge, quasi a commento di questa pretesa, "da dove, amico, risulta chiaro $(\delta \tilde{n} \lambda \mathrm{ov})$, qualcuno potrebbe dire, che il tuo filosofo discende da questo ordine di anime?". Al che Damascio risponde in modo molto prudente:

Io risponderò a questa domanda non in modo aggressivo, come se fossi in un tribunale, ma in modo piuttosto tranquillo; non con lo zelo di chi pretende la precisione assoluta, ma nel modo che è misurato per una biografia, esponendo solo quelle cose che io ritengo essere vere e che ho ascoltato dal mio maestro.

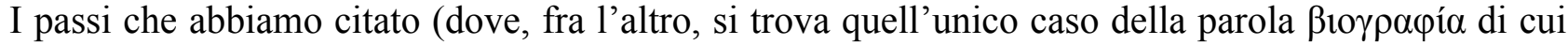
abbiamo detto) sono a mio parere decisivi per comprendere che tipo di opera è la Vita Isidori e qual è il suo scopo. 
Ciò che si legge nel fr. 7 di Fozio e che secondo la Suda Isidoro dice di sé non deve essere inteso tanto come l'attestazione della sua eccezionale singolarità, quasi fosse assimilabile a un semi-dio; ma piuttosto come documento della teoria neoplatonica della caduta dell'anima, che d'altro canto poteva contare sulla solida base testuale del Fedro platonico. Il riferimento a Platone è molto importante, perché sottrae la situazione descritta a qualunque inappropriata enfatizzazione: è del tutto normale per un platonico ritenere che il filosofo, ossia colui che secondo il Fedro conserva qui sulla terra un ricordo migliore dell'iperuranio (249e-250c), faccia dipendere il sapere che ha - tanto o poco che sia - dalla precedente conoscenza di una realtà sottratta alla generazione (e alla corruzione).

Ma ancora più importante è l'obiezione che si legge nel fr. 8 di Fozio. Qui, occorre avvertire, il contesto potrebbe essere diverso, perché non si parla tanto della discesa dell'anima, ma della sua appartenenza a una data stirpe; dunque, in questo caso, il riferimento platonico sarebbe non tanto al Fedro quanto al mito para-esiodeo delle razze che si legge nella Repubblica (414d-415c). Sia come sia, la differenza per noi non è rilevante, perché in entrambi i casi è in gioco la superiorità del filosofo, che a sua volta sottintende una maggiore affinità con il divino. L'aspetto interessante di questa obiezione, a mio avviso, è il semplice fatto che venga posta da un filosofo platonico, e che questi si senta in obbligo di rispondere. L'obiettore chiede che siano rese manifeste ( $\delta \hat{\eta} \lambda \mathrm{ov})$ le ragioni per cui il filosofo ha questa pretesa. Ma un filosofo platonico la cui anima fosse caduta nel mondo della generazione, e il cui unico scopo fosse quello di liberarsene il più presto possibile, perché dovrebbe trovare qualche interesse in tutto ciò? Da un lato egli è certo di essere decaduto da una situazione migliore, dall'altro non si vede, se la sua unica ambizione fosse quella di tornare quanto prima da dove è venuto, perché dovrebbe sentire il bisogno di giustificarsi. Con questo non voglio dire, si badi, che il desiderio del filosofo platonico di ritornare nell'iperuranio non sia reale. Un altro frammento di Fozio (cd. 242, 15) attesta che questo appunto era il desiderio di Isidoro; ma anche questa volta non abbiamo alcun bisogno di ascrivere questao atteggiamento alle deviazioni tarde del platonismo, visto che lo si ritrova interamente e chiaramente espresso già nel Fedone. Quello che voglio dire è che per Damascio il filosofo non è interamente proiettato nella dimensione ultratterena, ma intende il suo rapporto privilegiato con il divino, in modo del tutto platonico, come un titolo di credito per esercitare un determinato ruolo del mondo sensibile; poiché altrimenti non c'è ragione che egli si senta obbligato a mostrare a un profano la fondatezza delle sue credenziali. Il fatto che questa sia l'effettiva posizione di Damascio è confermato da un importate passo della Vita Isidori (Fozio, 242, 227), in cui egli mette in scena Isidoro che rimprovera lo scadente filosofo ateniese Egia (che aveva ridotto la filosofia a pratiche magico-teurgiche) nella maniera seguente:

Se è vero che è una cosa divina, come dici, la pratica teurgica..., questo lo dico anch'io; ma quelli che diventeranno degli dei prima sono degli uomini. Per questo anche Platone disse che presso gli uomini non è mai giunto un dono più grande della filosofia.

Qui la filosofia è intesa evidentemente alla luce del Simposio, in cui qualifica il sapere degli uomini

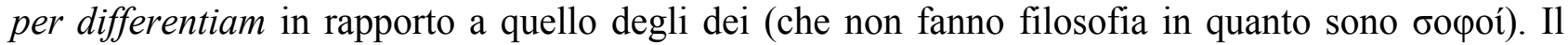
contatto del filosofo col divino non annulla, finché è uomo, la sua dimensione umana e non lo esenta dal compito di svolgere un ruolo direttivo nel mondo sensibile. Colui che sarà un domani pari agli dei, dunque sapiente, per ora è solo filosofo; per cui se vuole promuovere la superiorità del proprio sapere all'interno della dimensione mondana lo deve fare anzitutto sul piano umano della filo-sofia.

Assai significativa, infine, è la risposta singolarmente cauta di Damascio in Fozio 242, 8. Ben lungi dal replicare con sicumera o con arroganza (con l'arroganza, si intende, di chi si crede in possesso di una verità incontrovertibile, e dunque non è interessato a manifestare le sue ragioni), Isidoro risponde con la cautela e la calma di chi pretende per sé semplicemente di dire le cose che crede $(\pi 1 \sigma \tau \varepsilon v ́ \varepsilon \tau \alpha \imath)$ vere e quelle che ha udito direttamente dal suo maestro. Che è quanto dire: non esistono argomenti inconfutabili per certificare il sapere e la superiorità del fillosofo. Il filosofo, non 
diversamente dagli altri, è colui che crede che certe cose siano vere, e che evidentemente si impegna nell'esplicitare i fondamenti di questa sua credenza; quanto invece al filosofo che sia discepolo d'altri, e che si impegni a scriverne una biografia, avrà assolto il suo compito quando avrà riferito fedelmente quanto ha udito dal suo maestro.

A questo proposito alcune osservazioni a mio avviso si impongono. La prima riguarda la visibile somiglianza tra il contesto pagano qui descritto da Isidoro e quello cristiano. Gesù Cristo, non meno di Isidoro, dichiara di essere giunto qui da un luogo migliore (cfr. le frequenti allusioni al "padre mio che mi ha mandato", alla "casa del padre", ecc.). Anche i discepoli di Cristo, come quelli di Isidoro, ambiscono a dimostrare pubblicamente l'origine superiore del loro maestro; ma per fare questo non hanno altro mezzo che esporre le cose che credono essere vere ( $\pi \imath \tau \tau \varepsilon v ́ \omega$, in Damascio; ma ricordiamo che nei vangeli "fede" è $\pi i ́ \sigma \tau 1 \zeta)$ e ripetere fedelmente le parole del loro maestro. Con questo non intendo dire che Damacsio volesse paragonare direttamente Isidoro a Gesù. Come sappiamo, Damascio era ben consapevole che il suo maestro non aveva una personalità così distaccata. Ma in realtà è proprio questo il punto. Qui non importano, veramente, le persone concrete: cristiani o pagani ambiscono entrambi a un ruolo direttivo intellettuale e morale, pretendono entrambi che questo ruolo sia loro dovuto grazie a un contatto con il soprannaturale, ma per giustificare questa pretesa nessuno dei due ha molto di meglio che la fede sincera nelle cose che si ritengono vere e l'onesta esposizione di quanto udito da maestri che si suppongono autorevoli. L'idea è che se questo basa per i cristiani, dovrebbe bastare anche per i pagani. Perché pretendere di più? Come non vedere che $\mathrm{i}$ cristiani appoggiano la loro autorità a titoli non diversi da quelli che potrebbero avere anche i pagani?

Se quanto detto è plausibile comincia a poco a poco a delinearsi la figura del filosofo platonico ideale che Damascio dissemina, soprattutto nella sezione della sua opera specificamente dedicata a Isidoro, ma in parte anche tratteggiando altri personaggi. Anzitutto questo filosofo deve possedere, in qualche modo, un aggancio con il divino. Ciò è necessario per orientare la ricerca alla verità esattamente come era necessario in Platone che la dialettica fosse orientata dalla reminiscenza. Ma come dimostrare un nesso di questo genere? I mezzi di cui Damascio dichiara di disporre li conosciamo. Ripercorrendo la biografia di Isidoro racconterà le cose che ha buona ragione di credere vere. Così egli accenna, nella sua biografia, a sogni miracolosi (Fozio 242, 11; 25, Suda II 668,25 ) e al potere divinatorio che ne deriva (Fozio, fr, 12, 13, 14, 236; Suda III 627, 7; I 284, 8). E

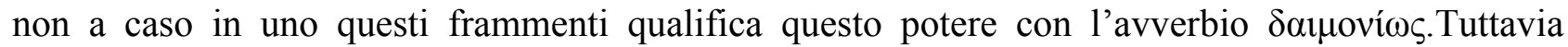
Damascio non sembra possedere in proposito elementi molto significativi, né dal punto di vista quantitativo né da quello qualitativo. E non basta. Nel passo in cui esprime la sua ammirazione per il grande potere divinatorio di Isidoro, dovuto ai favori a lui accordati dalla natura e dalla fortuna, non manca di aggiungere che quasi tutti gli alessandrini godono delle stesse predisposizioni. Questo passo ha il suo interesse in riferimento al dossier a cui abbiamo accennato all'inizio, ossia al filoallessadrismo della Vita Isidori in generale. Ma qui mi preme sottolineare un altro fatto. Damascio ha cura di precisare che un'importante prerogativa di Isidoro, per certi versi centrale nella sua biografia, non è in realtà una caratteristica sua personale. Per quanto uno possa essere ammirato dall'abilità divinatoria di Isidoro, è evidente che questa abilità dipende da un rapporto privilegiato con il divino. Ma questo rapporto non ha nulla di sorprendente: è anzi la normale condizione del filosofo platonico, certificata per primo (con tutta la chiarezza che si può desiderare) da Platone medesimo.

In altre parole sembra che per Damascio l'aggancio del sapere filosofico a una fonte soprannaturale sia in qualche modo scontato, ma proprio per questo non molto rilevante. In fondo anche i Cristiani pretendevano di attingere alla stessa fonte, e d'altra parte l'effettiva esistenza di questo contatto era praticamente impossibile da dimostrare. Damascio, come è ovvio, non si sottrae, ed anche su questo punto fa la sua parte, difendendo meglio che può le prerogative della tradizione a cui appartiene. E tuttavia appare nel complesso ben consapevole che gli esiti della partita si decidono altrove, sulle qualità umane del personaggio che prende a modello. Anzitutto si tratta di qualità psicologiche $\mathrm{e}$ morali, illustrate con una certa abbondanza in alcuni dei frammenti superstiti (Cfr. Fozio 242, 16, 
18, 19, 23, 28, 237, 307; Suda I 91, 26; 428, 27; 474, 16; II 273, 27; 298, 12; 337, 2; 525, 4;558, 4; 611, 1; III 443, 3: non ne parleremo qui). Ed è soprattutto sulla base di questi dati che Damascio può dire di Isidoro, in sintesi, che mostrava nelle sue opere l'elevato grado di somiglianza dell'uomo a dio di cui aveva parlato Pitagora (Suda, I 15, 10). Ma quando poi Damascio entra nel dettaglio si scopre che le qualità di Isidoro di cui si parla sono molto più umane che divine: in primo luogo la propensione a fare il bene e ad essere generoso con tutti, e dunque l'elevazione dell'anima al di sopra dei mali che affliggono questo mondo; in secondo luogo la capacità di preservare la dimensione corporea libera da ingiustizia e da empietà; infine, l'impegno nella vita pubblica nella misura del possibile. Come ben si vede, Damascio è consapevole che l'assimilazione al divino che l'uomo può conseguire è ben altra cosa dalla trasformazione vera e propria dell'uomo in dio.

Identico rapporto con la condizione umana reale, per quanto elevata ai massimi livelli delle sue possibilità, si riscontra anche a proposito delle qualità intellettuali. E' in apparenza sorprendente, in proposito, Fozio 242, 17, da cui si ricava che secondo Damascio Isidoro non aveva i sensi troppo sviluppati, e non possedeva nel complesso una memoria eccezionale (una caratteristica che viceversa Platone ritiene essenziale per il filosofo). In un primo tempo Damascio si trae d'impaccio dicendo che in questo modo il dio ha voluto mostrare che in Isidoro tutto dipendeva dalla sua anima e niente dal corpo. Ma proseguendo nel testo si fa strada anche un'altra idea: possedere una memoria profonda, ricca di moltissime teorie, ed essere capaci di sillogizzare indefinitamente, anche sulla base di un'acuta capacità percettiva, è proprio di filosofi che sono sapienti solo esteriormente, e che dunque sono poveri di quel vero sapere che si sviluppa all'interno, ossia nell'anima. Questo giudizio va di pari passo, del resto, con quanto si legge in Suda IV 164, 26, ossia che Isidoro non aveva letto molti libri, e con l'ulteriore precisazione in Fozio $(242,37)$, secondo cui Isidoro disprezzava l'erudizione libresca, che produce più ricchezza di opinioni che di intelligenza. La riprova della sua eccellenza interiore, viceversa, derivava dal fatto che aveva una straordinaria inventiva sia nel proporre lui stesso temi di discussione sia nel risolvere problemi proposti da altri (Fozio 242, 40), e che era abilissimo nel distinguere gli argomenti validi da quelli fallaci (Fozio 342, 41). Inoltre, precisa Damascio in un altro luogo, Isidoro era invincibile nella polemica dialettica; fino al punto da essere considerato come uno che soleva esprimersi un po' troppo liberamente (Fozio 242, 23), o addirittura litigioso di carattere (Fozio 242, 30). Unendo a questi elementi alcune precisazioni di Damascio, secondo cui la polemica di Isidoro era rivolta soprattutto verso il vizio morale (Fozio 242, 18, 19; Suda III 443, 3), ed era motivata dal suo ardente desiderio di correggere le azioni umane (Fozio 242, 20), ne viene fuori una specie di novello Socrate: nessuna particolare erudizione, nessuna buona qualità esterna, nessun particolare ornamento nel discorso (Damascio racconta sia che Isidoro disprezzava la retorica, 242, 35, sia che lui stesso non era dotato del dono della chiarezza espositiva, 242, 246), solo un'inesauribile capacità di discutere e di confutare, unita a una grande levatura morale, dimostrata sia con i fatti sia con i rimproveri che formulava a parole; e a questo si aggiunga che anche il Socrate di Platone si dichiarava, in qualche modo, a contatto con il divino.

In sintesi, per quanto abbiamo visto ora, il modello di filosofo platonico che andiamo cercando si ottiene sottraendo a Isidoro alcune idiosincratiche caratteristiche individuali. Non è ovviamente necessario che il filosofo platonico sia relativamente debole nei sensi, di memoria non troppo acuta e non particolarmente dotato quanto a capacità linguistico-espressive. Anzi, sarebbe ovviamente meglio che tali difetti non ci fossero. Ma proprio perché ci sono, permettono di intravedere che cosa per Damascio era essenziale; e forse non è nemmeno un caso che egli li abbia voluti sottolineare, perché in tal modo il lettore potesse osservare che ciò che è essenziale è anche riproducibile. Tolto il contatto col divino, che è una dotazione di partenza comune a tutti i sapienti, quello che poi conta sono le doti morali e un certo tipo di intelligenza, di stampo prevalentemente socratico, tutta interamente calata nell'interiorità della persona. Per Damascio, in altre parole, il sapiente platonico è colui che riesce sempre a cavare da se stesso, grazie all'elevatezza della sua anima, l'esempio concreto della virtù e il possesso delle argomentazioni migliori in vista della verità; e sono 
soprattutto queste eccellenti qualità umane che fanno dire di lui che è in qualche modo favorito dalla divinità.

Il modello socratico che abbiamo ricostruito ben doveva essere presente allo stesso Isidoro, in quanto sappiamo da Damascio che egli almeno una volta aveva preso le sue difese; ma lo ha fatto in modo troppo elevato e profondo perché i suoi discepoli potessero comprenderlo (Suda IV, 653, 26). Anche indipendentemente da questa citazione, sembra comunque ben plausibile che per un platonico del V-VI secolo d.C. l'interiorità socratica contenesse qualcosa di più di una straordinaria capacità argomentativa e confutatoria. Dunque il modello socratico deve essere indubbiamente orientato in chiave neoplatonica. Ma fino a che punto si spinge, Damascio, su questa strada? Per rispondere a questa domanda analizziamo brevemente, in chiusura, alcuni testi in cui Damascio spiega qual è il tipo di sapere posseduto dal suo ideale di filosofo.

Per una corretta conoscenza della realtà sono necessari, spiega Damascio (anche qui sulla scia di Platone) soprattutto tre qualità: amore, laboriosità, perspicacia ( $\alpha \gamma \chi i$ vot $\alpha$ ). Di queste, qualità, tuttavia, l'eros è la prima e la più importante, in quanto è il più formidabile indicatore e orientatore in vista di tutto quanto è bello e buono (Fozio 242, 31). Spiegando poi altrove la natura di queste tre qualità, Damascio chiarisce, nel più puro spirito di Platone, che esse possono essere utili all'interpretazione $(\delta i \alpha ́ \kappa \rho \imath \sigma \iota \varsigma)$ delle cose umane solo se sono intese come modelli divini, ossia come una laboriosità che eccede ogni discorso, come una perspicacia che va al di là delle comuni opinioni umane e come un eros che non si lascia mai distogliere dalle realtà intelligibili (Suda, III, 564, 1; IV, 516, 24). Come si vede, sia dal ruolo attribuito all'eros, sia dal modo in cui intende il modello divino (ossia come la massima misura consentita all'umano), l'ideale di Damascio è di nuovo molto platonico, e lontano dallo spiccato teocentrismo tipico di tanta tradizione neoplatonica. Quanto ora detto non contrasta né con l'idea che Damascio propone della storia della filosofia né con gli accenni che compaiono qua e là all'illuminazione divina. Per quanto riguarda il primo di questi due punti, Damascio individua anzitutto i filosofi che hanno limitato il loro interesse alle cose umane, come Aristotele e Crisippo; i quali, sia pure dotati di grande ingegno, per questo loro difetto non sono stati capaci di ottenere un sapere grande e degno di dio $(* * *)$. Per quanto riguarda Aristotele, in particolare, Damascio racconta che Isidoro lo avrebbe studiato all'inizio; ma poi se ne sarebbe distaccato perché si sarebbe accorto che Aristotele dimostrava fiducia "più all'intelletto necessario che a quello appropriato" (***), e che era più attento agli aspetti tecnici che a quelli propriamente divini. Quello che Damascio vuol dire, in sostanza, è che Aristotele ha usato l'intelletto e la ragione in modo riduttivo, vincolandoli alla necessità della dimostrazione, invece che alla scoperta di oggetti a essi appropriati. Tutto questo, di nuovo, corrisponde al normale sentimento platonico, secondo cui gli oggetti che sono davvero oggetto primario di desiderio e di conoscenza hanno carattere divino e ultrasensibile. Ed è sostanzialmente platonica anche l'idea che la causa che in ultima analisi produce la conoscenza non sia da ricercarsi nelle particolari caratteristiche delle facoltà umane (siano esse la $\varphi \alpha v \tau \alpha \sigma i ́ \alpha$, la $\delta o ́ \xi \alpha$ o anche la $\delta$ ióvol $\alpha$ ), ma in una illuminazione preliminare che rende la conoscenza possibile. In altre parole, senza un momento intuitivo preliminare, nessuna facoltà conoscitiva, nemmeno la $\delta$ ióvoı $\alpha$, sarebbe in grado di produrre il sapere. Ma ciò corrisponde di nuovo, senza troppe sbavature, alla reminiscenza platonica, ossia a quel preliminare sapere intuitivo che in Platone è condizione di possibilità del pensiero discorsivo ${ }^{11}$. Altro problema è capire se per Damascio questa illuminazione funge solo da sfondo per rendere

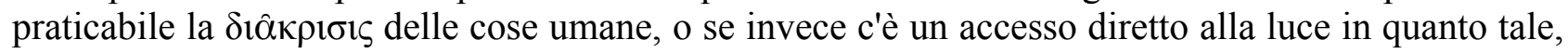
capace di mettere in mora fin da adesso la limitatezza della condizione umana. Se stiamo a Isidoro, e alle regole della biografia, Damascio non può che ripetere quel lo che ha udito dal suo maestro:

Egli diceva che l'anima, durante le sacre preghiere, davanti al mare intero del divino, da principio si raccoglie in se stessa separandosi dal corpo, poi si distacca dai suoi comportamenti propri e si allontana dai pensieri

\footnotetext{
${ }^{11}$ Per queta interpretazione della reminiscenza v. Trabattoni 2011, XXXIV-XLVIII.
} 
razionali per dirigersi verso quelli che sono congeneri all'intelletto; infine, in terzo luogo, è posseduta dal divino e si trasferisce in una serenità inusuale, conforme a dio e non umana (Fozio, 242, 240).

Questo, dunque è quanto diceva Isidoro. Damascio lo ripete, con sincera fiducia, così come dimostra di credere al soprannaturale, alle profezie, alla teurgia. Dunque l'incidente felice (in questo caso) che taglia le gambe alla filosofia è sempre possibile. Ma Damascio fa ben capire che non si tratta della via maestra. Gli uomini sapienti si assimileranno completamente a dio solo dopo la morte. Certo, a individui particolarmente dotati è in questo mondo può essere concesso qualche assaggio. Ma si tratta di eventi accidentali e sporadici, che poco hanno a che fare con l'ordinaria dimensione del filosofo e con la sua auspicata riproducibilità. L'assistenza divina, se intesa in senso generico come orizzonte di riferimento e come condizione di possibilità del sapere teorico e della salvezza pratica, è concessa a tutti i sapienti. Ma questa assistenza divina, per chi come noi guarda il mondo dal basso, può essere solidamente e oggettivamente certificata (o resa chiara: cfr. l'uso di $\delta \hat{\eta} \lambda$ ov in 242,8$)$ solo in base a considerazioni che partono anch'esse dal basso: ciò che apparenta l'uomo al divino già in questo mondo è la sua spinta inesaurible all'autosuperamento (eros), che si manifesta nella pratica della virtù e nella ricerca del sapere. La divinizzazione dell'uomo, finché l'uomo è uomo, è contenuta tutta all'interno della sua umanità ${ }^{12}$.

\section{Bibliografia}

Asmus, J. R. (1909-1910) : "Zur Rekonstruktion von Damascius' Leben des Isidorus", Byzantinische Zeitschrift 18, 424-480; 19, 265-284.

Asmus, J. R. (1910) : "Der Kyniker Sallustius bei Damascius", in Neue Jahrbücher für das klassische Altertum, Geschichte und deutsche Literatur, 25, 504-522.

Asmus, J. R. (1911) : Das Leben des Philosophen Isidoros von Damaskios aus Damaskos, Lepzig.

Athanassiadi, P. (1999) : Damascius. The Philosophical History. Text with translation and notes, Athènes.

Goulet, R. (1998) : Histoire et mistère. Les vies de philosophes dans l'antiquité tardive, in W. W. Ehlers (éd.), La biographie antique, Fondation Hardt, Entretiens sur l'antiquité classique, Vandœvre-Geneve.

Goulet, R. (2001) : Études sur les Vies de philosophes de l'antiquité tardive. Diogène Laërce, Porphyre de Tyr, Eunape de Sardes, Paris.

Hoffmann, Ph. (1994) Damascius, in R. Goulet (éd.) Dictionnaire des philosophes antiques, Bd. 2 , Paris 541-593.

Momigliano. A. (1974) : Lo sviluppo della biografia greca.

O' Meara, D. (2006) : "Patterns of Perfection in Damascius' 'Life of Isidore'", Phronesis 51, 74-90.

Trabattoni, F. (1985) : "Per una biografia di Damascio", Rivista di Storia della Filosofia 41, 179201

Trabattoni, F. (2003) : Filosofia e dialettica in Damascio, in M. Barbanti, G. Giardina, P. Manganaro, ENWSIS KAI FILIA. Unione e amicizia. Omaggio a Francesco Romano, Catania, 477-494.

Trabattoni, F. (2011) (cur.) : Platone, Fedone, Torino.

Zintzen, C. (1967) (ed.) : [Damascii] Vita Isidori reliquia, Hildesheim.

\footnotetext{
${ }^{12}$ Per un approfondimendo di questa immagine prevalentemente "umanistica", per dir così, della filosofia di Damascio, rinvio a Trabattoni 2003.
} 